\title{
Glutaraldehyde cross-linking of amniotic membranes affects their nanofibrous structures and limbal epithelial cell culture characteristics
}

This article was published in the following Dove Press journal:

International Journal of Nanomedicine

30 October 2013

Number of times this article has been viewed

Jui-Yang Lai ${ }^{1-3}$

David Hui-Kang Ma ${ }^{4,5}$

'Institute of Biochemical and Biomedical Engineering, ${ }^{2}$ Biomedical Engineering Research Center, ${ }^{3}$ Molecular Medicine Research Center, Chang Gung University, Taoyuan, Taiwan; ${ }^{4}$ Limbal Stem Cell Laboratory, Department of Ophthalmology, Chang Gung Memorial Hospital, Taoyuan, Taiwan; ${ }^{5}$ Department of Chinese Medicine, Chang Gung University, Taoyuan, Taiwan
Correspondence: Jui-Yang Lai Institute of Biochemical and Biomedical Engineering, Chang Gung University, 259, Wen-Hwa Ist Road, Kwei-Shan, Taoyuan, Taiwan 33302

Tel +88632118800 ext 3598

Fax +886 32118668

Email jylai@mail.cgu.edu.tw
Abstract: Given that the cells can sense nanometer dimensions, the chemical cross-linkingmediated alteration in fibrillar structure of collagenous tissue scaffolds is critical to determining their cell culture performances. This article explores, for the first time, the effect of nanofibrous structure of glutaraldehyde (GTA) cross-linked amniotic membrane (AM) on limbal epithelial cell (LEC) cultivation. Results of ninhydrin assays demonstrated that the amount of new crosslinks formed between the collagen chains is significantly increased with increasing the crosslinking time from 1 to 24 hours. By transmission electron microscopy, the AM treated with GTA for a longer duration exhibited a greater extent of molecular aggregation, thereby leading to a considerable increase in nanofiber diameter and resistance against collagenase degradation. In vitro biocompatibility studies showed that the samples cross-linked with GTA for 24 hours are not well-tolerated by the human corneal epithelial cell cultures. When the treatment duration is less than 6 hours, the biological tissues cross-linked with GTA for a longer time may cause slight reductions in 3-(4,5-dimethylthiazol-2-yl)-5-(3-carboxymethoxyphenyl)-2-(4-sulfophenyl)$2 \mathrm{H}$-tetrazolium, inner salt, and anti-inflammatory activities. Nevertheless, significant collagen molecular aggregation also enhances the stemness gene expression, indicating a high ability of these AM matrices to preserve the progenitors of LECs in vitro. It is concluded that GTA cross-linking of collagenous tissue materials may affect their nanofibrous structures and corneal epithelial stem cell culture characteristics. The AM treated with GTA for 6 hours holds promise for use as a niche for the expansion and transplantation of limbal epithelial progenitor cells.

Keywords: glutaraldehyde, amniotic membrane, nanofibrous structure, limbal epithelial cell, stemness

\section{Introduction}

Tissue engineering is an interdisciplinary field that aims to regenerate natural tissues and organs using cells, biomaterial scaffolds, and signaling molecules. ${ }^{1}$ As a wellknown biomaterial, the amniotic membrane (AM) is the innermost layer of the placenta that has been adopted in clinical practice for many years. ${ }^{2}$ This biological tissue contains several growth factors and is featured with low immunogenicity and anti-inflammatory, antiangiogenic, and antiscarring properties. ${ }^{3}$ To avoid the postoperative complications associated with the use of gastrointestinal segments for bladder reconstruction, AM is considered as an alternative material for augmentation. ${ }^{4}$ The good biocompatibility of AM also allows this biomaterial to be used as an attractive dermal matrix for the construction of living skin equivalents in the treatment of burn wounds, skin defects, and chronic wounds..$^{5}$ In ophthalmology, AM materials have been demonstrated to provide a unique microenvironment for supporting the growth and maintenance of 
corneal epithelial progenitor cells. ${ }^{6} \mathrm{~A}$ technique based on the transplantation of limbal epithelial cells (LECs) cultivated on the AM matrices may restore vision for patients afflicted with unilateral corneal stem cell deficiency. ${ }^{7}$

For AM grafts, the collagen nanofibers in these biological tissues are usually stabilized by peptide bridges. It has been documented that corneal diseases can increase the action of tissue collagenase, ${ }^{8}$ thereby accelerating the degradation of AM matrices after surgical transplantation. In our laboratory, to further improve the material stability against enzymatic cleavage, the AM is cross-linked by exogenous carbodiimide. ${ }^{9}$ After the formation of numerous amide linkages between the carboxyl and amino groups in AM collagen, the chemically modified proteinaceous matrices exhibit enhanced resistance to collagenase digestion, probably because of the protective effect caused by the aggregation of collagen nanofibers. However, according to our observations, the treatment of AM with carbodiimide has limited cross-linking efficiency. For the carbodiimide-treated AM samples with a saturated cross-linking index of around $45 \%$, approximately $20 \%$ of weight loss is noted during the degradation period from 2 to 4 weeks, and the residual tissues gradually degrade with time. Therefore, the use of an alternative chemical cross-linker for AM modification is highly desired.

Carbodiimide is a zero-length cross-linker capable of inducing amide bond formation between the amino acid residues in proteins without added bridging moieties. ${ }^{10}$ In contrast, glutaraldehyde (GTA) (ie, a non-zero-length cross-linker that directly joins the molecules together) may participate in the formation of covalent bonds (Schiff bases). ${ }^{11}$ The construction of a more rigid biomacromolecular network structure is achieved by the incorporation of cross-links between polypeptide chains. Although chemical modification of biomaterials with GTA may pose a risk for toxicity, ${ }^{12,13}$ this cross-linking treatment is also found to be clinically acceptable for the fabrication and implantation of bioprosthesis. ${ }^{14}$ Furthermore, Matsuda et al have reported that the aldehyde groups introduced in the cross-linked biopolymers can be quenched with glycine to reduce the cytotoxicity of chemical compounds. ${ }^{15}$ Our previous study has also shown that human retinal pigment epithelial cell viability is significantly increased for GTA cross-linked gelatin samples with glycine treatment compared with their counterparts without glycine treatment. ${ }^{16}$ Therefore, in this study, the AM materials following GTA cross-linking are placed in glycine aqueous solution for the capping of residual aldehyde groups.

It has been reported that the mechanical stability of dermal sheep collagen strongly depends on the aggregated structure of nanofibers and the spatial arrangement of the fiber bundles. ${ }^{17}$ Given that the formation of cross-links in the carbodiimidetreated AM samples leads to significant aggregation of tropocollagen molecules and collagen microfibrils, ${ }^{9}$ it may further affect the stability of chemically modified biological tissues. As reported in the literature, the AM materials have been treated by GTA cross-linking. ${ }^{18,19}$ However, these previous studies only examine the cross-linking treatment on the biomechanical and biodegradation properties of AM matrices but do not provide direct insights into the collagen molecular structure and its influence on cell culture characteristics. In the current article, we aim to clarify this issue. After modification with GTA for varying cross-linking times, the AM samples were studied by determinations of cross-linking degree, nanofibrous structure, in vitro degradability, cytocompatibility, anti-inflammatory activity, and stemness gene expression. To the best of our knowledge, the role of GTA cross-linkingmediated nanoscale structure in the cell culture performance of the AM has not been investigated.

\section{Materials and methods Materials}

This study followed the tenets of the Declaration of Helsinki involving human subjects and received approval from the institutional review board of Chang Gung Memorial Hospital. With informed consent, human AM tissues (ie, the innermost layer of the placental membranes) were obtained at the time of elective cesarean section, from mothers when human immunodeficiency virus, syphilis, and hepatitis $\mathrm{B}$ and $\mathrm{C}$ had been excluded by serologic tests. The separation of AM was carried out, using blunt dissection to cut the tissue samples approximately $2 \mathrm{~cm}$ from the placental disc. The average thickness of AM samples used in this work was $100 \mu \mathrm{m}$. 1-ethyl-3-(3-dimethylaminopropyl) carbodiimide hydrochloride (EDC), GTA, ninhydrin reagent, collagenase (type I Clostridium histolyticum; EC 3.4.24.3), and lipopolysaccharide (LPS) were purchased from SigmaAldrich (St Louis, MO, USA). N-Hydroxysuccinimide (NHS) was supplied by Acros Organics (Geel, Belgium). Dimethyl sulfoxide (DMSO) was obtained from JT Baker Inc (Phillipsburg, NJ, USA). Deionized water used was purified with a Milli-Q system (Millipore, Bedford, MA, USA). Phosphate-buffered saline (PBS, $\mathrm{pH}$ 7.4) was purchased from Biochrom AG (Berlin, Germany). Balanced salt solution (BSS, pH 7.4) was obtained from Alcon Laboratories (Fort Worth, TX, USA). 2-( $N$-morpholino)ethanesulfonic acid (MES; JT Baker Inc) was dissolved in deionized water to form a $0.05 \mathrm{M}$ buffer solution ( $\mathrm{pH}$ 5.5). Dispase II was 
purchased from Roche Diagnostics (Indianapolis, IN, USA). FNC Coating Mix (ie, a fibronectin/collagen mixture) was obtained from AthenaES (Baltimore, MD, USA). Dulbecco's Modified Eagle's Medium (DMEM), keratinocyte serumfree medium (KSFM), Ham's F-12 nutrient mixture (Ham's F-12), gentamicin, trypsin-ethylenediaminetetraacetic acid (EDTA), and TRIzol reagent were purchased from Gibco-BRL (Grand Island, NY, USA). Fetal bovine serum (FBS) and the antibiotic/antimycotic (A/A) solution $(10,000 \mathrm{U} / \mathrm{mL}$ penicillin, $10 \mathrm{mg} / \mathrm{mL}$ streptomycin, and 25 $\mu \mathrm{g} / \mathrm{mL}$ amphotericin B) were obtained from Biological Industries (Kibbutz Beit Haemek, Israel). 24-well tissue culture polystyrene (TCPS) plates (Falcon 353047) were purchased from Becton Dickinson Labware (Franklin Lakes, NJ, USA). All the other chemicals were of reagent grade and used as received without further purification.

\section{Preparation of chemically cross-linked amniotic membranes}

The AM samples were aseptically washed three times with PBS containing $1 \% \mathrm{~A} / \mathrm{A}$ solution and $50 \mu \mathrm{g} / \mathrm{mL}$ gentamicin according to the protocols reported previously. ${ }^{20}$ The membranes were immersed with sequential concentrations of DMSO, followed by freezing and storing at $-80^{\circ} \mathrm{C}$ in DMEM containing 50\% glycerol. After a further incubation with $0.02 \%$ EDTA at $37^{\circ} \mathrm{C}$, the AM was denuded of its amniotic epithelial cells by gentle scraping. For carbodiimide cross-linking, the membranes were immersed in $30 \mathrm{~mL}$ MES buffer containing EDC and NHS under gentle shaking. The cross-linker concentration was fixed at $0.05 \mathrm{mmol} \mathrm{EDC} / \mathrm{mg}$ AM. In addition, a 5:1 EDC-to-NHS molar ratio was used for all cross-linking. The cross-linking reaction was allowed to proceed at $25^{\circ} \mathrm{C}$ for 6 hours. The resulting membranes were thoroughly washed with deionized water to remove excess EDC and urea byproduct. In contrast, the AM samples were treated with GTA by immersing the membranes in $30 \mathrm{~mL}$ PBS containing cross-linking agent under gentle shaking. The cross-linker concentration was fixed at $0.05 \mathrm{mmol} \mathrm{GTA} / \mathrm{mg}$ AM. The cross-linking reaction was allowed to proceed at $25^{\circ} \mathrm{C}$ for $1-24$ hours, and the resulting membranes were then placed in $100 \mathrm{mM}$ glycine aqueous solution at $37^{\circ} \mathrm{C}$ for 1 hour to block unreacted residual GTA. In this study, the biological tissue materials modified with EDC and GTA for 6 hours were respectively designated as ET6 and GT6.

\section{Cross-linking degree}

The ninhydrin assay was used to determine the amount of free amino groups of each AM. The test sample was weighed and heated with a ninhydrin solution for 20 minutes. After the test solution was cooled to room temperature and diluted in 95\% ethanol, the optical absorbance of the solution was recorded with an ultraviolet-visible spectrophotometer (Thermo Scientific, Waltham, MA, USA) at $570 \mathrm{~nm}$, using glycine at various known concentrations as standard. ${ }^{21}$ The amount of free amino groups in the AM before $\left(C_{\mathrm{b}}\right)$ and after $\left(C_{\mathrm{a}}\right)$ cross-linking is proportional to the optical absorbance of the solution. The degree of cross-linking of the AM was calculated as cross-linking index $(\%)=\left(\left[C_{\mathrm{b}}-C_{\mathrm{a}}\right] / C_{\mathrm{b}}\right) \times 100$. Results were the average of six independent measurements.

\section{Nanostructure}

Specimens were processed for transmission electron microscopy (TEM) studies as described previously. ${ }^{22}$ Various preparations of AM were fixed and washed in $0.1 \mathrm{M}$ cacodylic acid buffer ( $\mathrm{pH} 7.4$ ). The samples were postfixed in $1 \%$ osmium tetroxide and dehydrated in a graded series of ethanol solutions. The tissue specimens were then infiltrated with Spurr's resin through a resin:ethanol series of 1:1, 3:1, 100\% Spurr's. After infiltration, the samples were cut into blocks of a width of $1 \mathrm{~mm}$, placed in flat embedding molds, and polymerized at $70^{\circ} \mathrm{C}$ overnight. Ultrathin sections were cut using a diamond knife on a Reichert Ultracut S microtome (Leica Microsystems, Wetzlar, Germany), stained with 2\% uranyl acetate, and visualized using a JEM-1230 TEM (Jeol, Tokyo, Japan). For each tissue sample, five randomly chosen fields were counted at 30,000 $\times$ magnification, and the diameter of 200 collagen fibrils was measured with an ocular micrometer. Results were the average of three independent experiments.

\section{In vitro degradability}

To measure the extent of degradation, each test AM $\left(1 \times 1 \mathrm{~cm}^{2}\right)$ was first dried to constant weight $\left(W_{\mathrm{i}}\right)$ in vacuo and was immersed in $1 \mathrm{~mL} \mathrm{BSS}$ containing $12 \mu \mathrm{g}$ collagenase at $37^{\circ} \mathrm{C}$ with reciprocal shaking $(50 \mathrm{rpm})$ in a thermostatically controlled water bath. Degradation medium was replaced weekly with fresh buffer solution containing the same concentration of enzyme. ${ }^{23}$ After 4 weeks, the membrane samples were taken out and washed with deionized water. The degraded samples were further dried in vacuo and weighed to determine the dry weight $\left(W_{\mathrm{d}}\right)$. The percentage of weight remaining was calculated as $\left(W_{\mathrm{d}} / W_{\mathrm{i}}\right) \times 100$. Results were the average of four independent measurements.

\section{In vitro biocompatibility}

In this study, HCE-2 cells, a human corneal epithelial cell line (ATCC No CRL-11135), were purchased from the American 
Type Culture Collection (Manassas, VA, USA). The cells were seeded on tissue culture plastics precoated with FNC Coating Mix and maintained in regular growth medium containing KSFM, $0.05 \mathrm{mg} / \mathrm{mL}$ bovine pituitary extract, $5 \mathrm{ng} /$ $\mathrm{mL}$ epidermal growth factor, $500 \mathrm{ng} / \mathrm{mL}$ hydrocortisone, and $0.005 \mathrm{mg} / \mathrm{mL}$ insulin. Cultures were incubated in a humidified atmosphere of $5 \% \mathrm{CO}_{2}$ at $37^{\circ} \mathrm{C}$. The medium was changed twice a week. Cells were subcultured by trypsinization at a split ratio of 1:3.

The in vitro biocompatibility evaluation of the AM samples was conducted in adaptation from the ISO10993-5 standard test method. ${ }^{24} \mathrm{~A}$ single extract of the test article was prepared using regular growth medium. The extracts were obtained by incubation of the ultraviolet-sterilized materials with culture medium at $37^{\circ} \mathrm{C}$ for 24 hours with an extraction ratio of $0.2 \mathrm{~g} / \mathrm{mL}$. Each test extract was then placed onto HCE-2 cell cultures with a seeding density of $5 \times 10^{4}$ cells/well. After a 3 -day incubation at $37^{\circ} \mathrm{C}$ in the presence of $5 \% \mathrm{CO}_{2}$, the qualitative and quantitative assays were performed to examine the cellular responses to chemically cross-linked AM. The cells in regular growth medium without contacting material samples served as control groups.

\section{Cell viability assays}

Cell viability was determined using a membrane integrity assay, the Live/Dead Viability/Cytotoxicity Kit (Molecular Probes, Eugene, OR, USA), which contains calcein acetoxymethyl and ethidium homodimer 1 . It depends on the intracellular esterase activity to identify the living cells, which cleaves the calcein acetoxymethyl to produce a green fluorescence. In dead cells, ethidium homodimer 1 can easily pass through the damaged cell membranes to bind to the nucleic acids, yielding a red fluorescence. After washing three times with PBS, the cultures were stained with a working solution consisting of $2 \mu \mathrm{L}$ ethidium homodimer 1 , $1 \mathrm{~mL}$ PBS, and $0.5 \mu \mathrm{L}$ calcein acetoxymethyl. Under fluorescence microscopy (Axiovert 200M; Carl Zeiss, Jena, Germany), the live and dead cells in three fields were counted at $100 \times$ magnification. All experiments were performed in triplicate, and the viability of the HCE-2 cell cultures was expressed as the average ratio of live cells to the total number of cells in these nine different areas.

\section{Cell proliferation assays}

Cell growth was estimated using the CellTiter 96 Aqueous Non-Radioactive Cell Proliferation Assay (Promega, Madison, WI, USA), in which 3-(4,5-dimethylthiazol-2-yl)-5-(3carboxymethoxyphenyl)-2-(4-sulfophenyl)-2H-tetrazolium, inner salt, tetrazolium compound is bioreduced by cells to form a water-soluble colored formazan. The amount of colored product is proportional to the number of metabolically active cells. One hundred microliters of the combined MTS/ phenazine methosulfate $(20: 1)$ reagent was added to each well of the 24-well plate and incubated for 3 hours at $37^{\circ} \mathrm{C}$ in a $\mathrm{CO}_{2}$ incubator. The data of absorbance readings at $490 \mathrm{~nm}$ were measured using the Multiskan Spectrum Microplate Spectrophotometer (Thermo Labsystems, Vantaa, Finland). All experiments were performed in quadruplicate, and the results were expressed as relative MTS activity when compared with control groups.

\section{Proinflammatory gene expression analyses}

Total RNA was isolated from cells with TRIzol reagent according to the manufacturer's procedure. Reverse transcription of the extracted RNA $(1 \mu \mathrm{g})$ was performed using ImProm-II ${ }^{\mathrm{TM}}$ (Promega) and Oligo(dT) ${ }_{15}$ primers (Promega). The primers used to amplify the human interleukin $6(I L-\sigma)$ complementary DNA (cDNA) were 5'-CCACTCACCTCTTCAGAACGAA-3' (sense) and 5'-GGCAAGTCTCCTCATTGAATCC-3' (antisense). The sequences of the primer pair used to amplify the internal control cDNA, glyceraldehyde-3-phosphate dehydrogenase $(G A P D H)$, were 5'-TGGTATCGTGGAAGGACTCATGAC-3' (sense) and 5'-ATGCCAGTGAGCTTCCCGTTCAGC-3' (antisense). Quantitative real-time reverse transcription polymerase chain reaction (RT-PCR) was performed on a Light-Cycler ${ }^{\circledR}$ instrument (Roche Diagnostics), according to the manufacturer's instructions, with FastStart DNA Master SYBR Green I reagent (Roche Diagnostics). Each sample was determined in triplicate, and the gene expression results were normalized to the level of glyceraldehyde 3-phosphate dehydrogenase $(G A P D H)$ mRNA.

\section{Anti-inflammatory activity}

HCE-2 cells $\left(5 \times 10^{4}\right.$ cells/well $)$ were seeded in 24-well plates containing regular growth medium and incubated overnight. For LPS stimulation, the medium was replaced with the fresh medium containing $1 \mu \mathrm{g} / \mathrm{mL}$ because high dosages of LPS $(>1 \mu \mathrm{g} / \mathrm{mL})$ caused cell death and low dosages $(<0.01 \mu \mathrm{g} / \mathrm{mL})$ showed little stimulation of cells. ${ }^{25}$ Using cell culture inserts (Falcon 3095, Becton Dickinson Labware), each well of a 24-well plate was divided into two compartments. A sterilized membrane sample was placed into the inner well of the double-chamber system to examine the LPS-stimulated cultures after exposure to 
chemically cross-linked AM materials. Unstimulated and LPS-stimulated HCE-2 cells without contacting the test samples served as the negative control (NC) and positive control (PC) groups, respectively.

After 3 days of incubation, the release of tumor necrosis factor- $\alpha(\mathrm{TNF}-\alpha)$ from cultivated cells into the conditioned medium was detected by the Quantikine enzyme-linked immunosorbent assay kit (R\&D Systems, Minneapolis, MN, USA) specific for human TNF- $\alpha$. Aliquots of the supernatant from each well were collected, and cytokine bioassays were performed according to the manufacturer's instructions. Photometric readings at $450 \mathrm{~nm}$ were measured using the Multiskan Spectrum Microplate Spectrophotometer (Thermo Labsystems). Results were expressed as picograms per milliliter. All experiments were conducted in quadruplicate.

\section{Stemness gene expression}

The rabbit corneoscleral rims were used to culture LECs. To disperse the cells, the corneoscleral rims were treated with Dispase II at $37^{\circ} \mathrm{C}$, followed by incubation with trypsinEDTA solution. The cultures were maintained with supplemental hormonal epithelial medium, which was made of an equal volume of hydroxyethyl piperazineethanesulfonic acid-buffered DMEM containing bicarbonate and Ham's F-12, 0.5\% DMSO, $2 \mathrm{ng} / \mathrm{mL}$ mouse epidermal growth factor, $5 \mu \mathrm{g} / \mathrm{mL}$ insulin, $5 \mu \mathrm{g} / \mathrm{mL}$ transferrin, $5 \mathrm{ng} / \mathrm{mL}$ selenium, $0.5 \mu \mathrm{g} / \mathrm{mL}$ hydrocortisone, $30 \mathrm{ng} / \mathrm{mL}$ cholera toxin $\mathrm{A}$ subunit, 5\% FBS, $50 \mu \mathrm{g} / \mathrm{mL}$ gentamicin, and $1.25 \mu \mathrm{g} / \mathrm{mL}$ amphotericin B.

Rabbit LECs $\left(5 \times 10^{4}\right.$ cells/well $)$ were seeded into 24-well TCPS plates (control groups) and various sterilized AM materials. After incubation in supplemental hormonal epithelial medium at $37^{\circ} \mathrm{C}$ for 5 days, the total RNA was isolated from cells and the mRNA expressions were quantified by real-time RT-PCR. The primers used to amplify the rabbit $A B C G 2$ cDNA were 5'-GAGAGCTGGGTCTGGAAAAAGT-3' (sense) and 5'-ATTCTTTTCAGGAGCAGAAGGA-3' (antisense). The sequences of the primer pair used to amplify the internal control cDNA, GAPDH, were 5'-TTGCCCTCAATGACCACTTTG-3' (sense) and 5'-TTACTCCTTGGAGGCCATGTG-3' (antisense). Each sample was determined in triplicate, and the gene expression results were normalized to the level of GAPDH mRNA.

\section{Statistical analysis}

Results were expressed as mean \pm standard deviation. Comparative studies of means were performed using one-way analysis of variance. Significance was accepted with $P<0.05$.

\section{Results and discussion Cross-linking degree}

Here, the cross-linking degrees of chemically modified collagenous tissues were evaluated by ninhydrin assays, given that the reaction with GTA involves the consumption of free amino groups of lysine or hydroxylysine amino acid residues of the polypeptide chains. Figure 1 shows the cross-linking index of various AM samples. In the ET6 groups, the crosslinking index was $46.1 \% \pm 1.8 \%$, which was not significantly different from those prepared by the cross-linking with carbodiimide for 24 hours $(P>0.05){ }^{9}$ The results indicate that during the EDC/NHS reaction, 6 hours of treatment time is sufficient to achieve saturated cross-linking. In contrast, for the GTA cross-linked AM materials, the cross-linking index in the GT1, GT6, and GT24 groups was $8.5 \% \pm 2.7 \%$, $32.3 \% \pm 2.0 \%$, and $68.8 \% \pm 2.4 \%$, respectively, suggesting that the amount of new cross-links formed between the collagen chains is significantly increased with increasing reaction time from 1 to 24 hours $(P<0.05)$. These findings are consistent with our previous report on chemical modification of porous gelatin hydrogels by demonstrating that longer treatment duration may lead to a greater extent of cross-linking. ${ }^{26}$ Interestingly, at the same cross-linking time (ie, 6 hours), the efficiency of GTA treatment is much lower than in the case of carbodiimide methods. GTA is known to be very reactive. ${ }^{27}$ At the initial stage, the cross-linking reaction may proceed too quickly to reduce the mobility of protein molecules and to form surface barriers to cross-linker permeation. Nevertheless, after the GTA diffusion into the AM matrix with time, these effects are weakened because of continued generation of cross-links by collision between the collagen and cross-linker molecules.

\section{Nanostructure}

In the research field of nanofibrous biomaterial modification, TEM is usually used to identify the ultrastructure. It has been well-recognized that the collagen molecules are able to assemble into filamentous collagen fibrils and subsequently aggregate to form collagen fibers. ${ }^{28}$ The effect of chemical modification on AM ultrastructural alteration was observed by TEM. Figure 2A shows representative images of various cross-linked AM samples. In the ET6 groups, the carbodiimide-treated biological tissue materials demonstrated a pattern of aggregated fibrils, indicating the role of chemical cross-linker in the modulation of fibrillar 


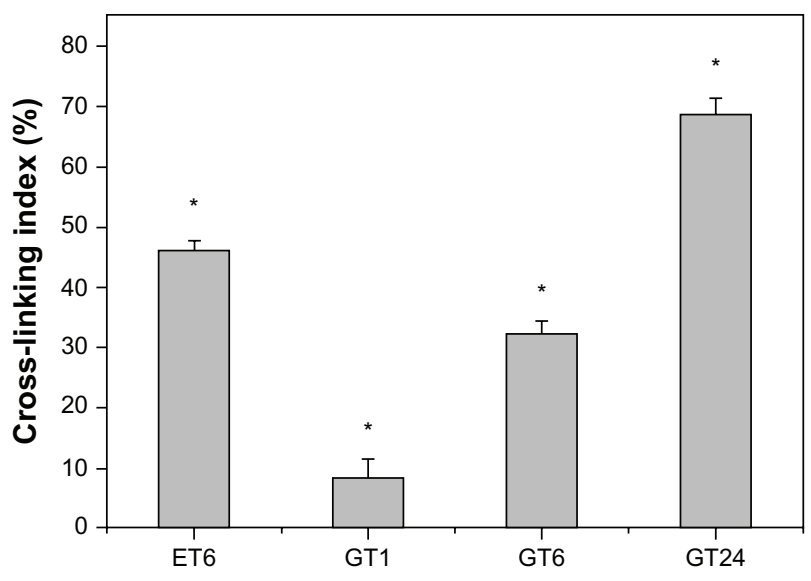

Figure I Cross-linking index of various amniotic membrane samples. Values are mean \pm standard deviation $(n=6)$.

Note: $* P<0.05$ versus all groups.

Abbreviations: EDC, I-ethyl-3-(3-dimethylaminopropyl) carbodiimide hydrochloride; ET6, biological tissue materials modified with EDC for 6 hours; GTA, glutaraldehyde; GTI, biological tissue materials modified with GTA for I hour; GT6, biological tissue materials modified with GTA for 6 hours; GT24, biological tissue materials modified with GTA for 24 hours.
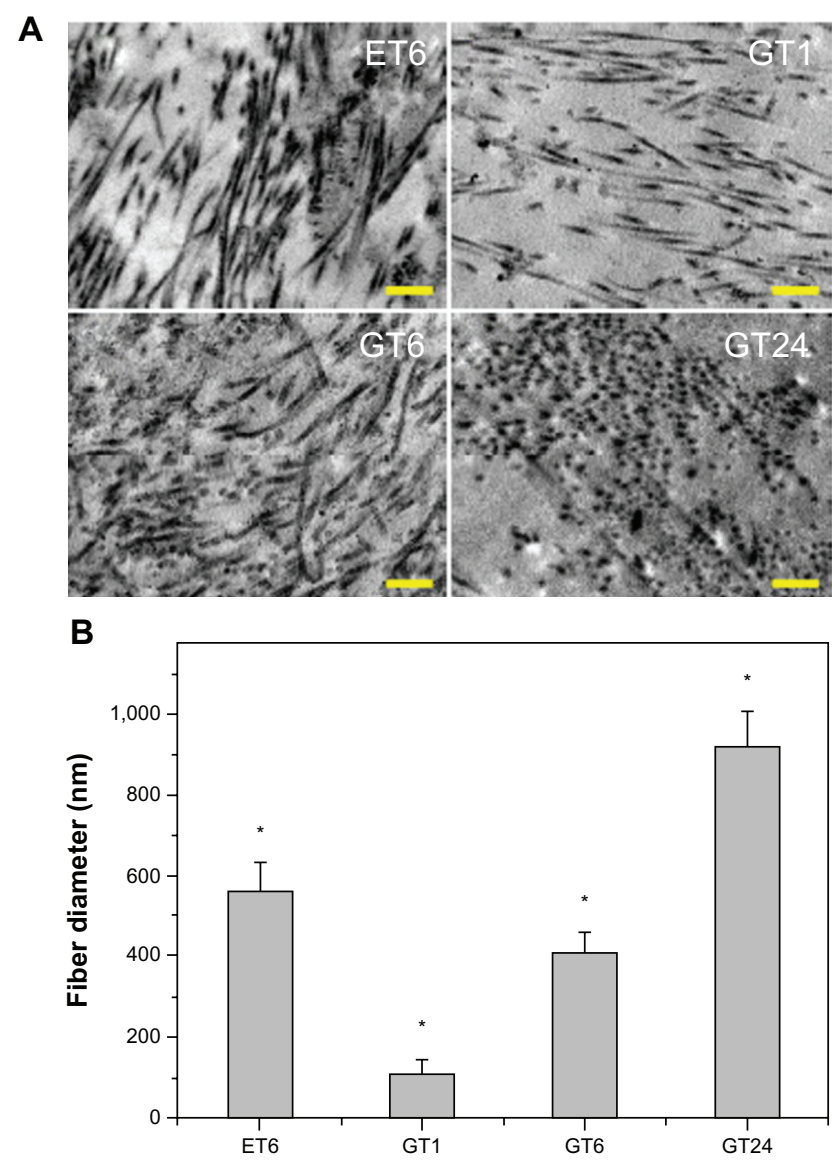

Figure 2 (A) Representative transmission electron microscopy images of various amniotic membrane samples. (B) Fiber diameter of membrane structure.

Notes: Scale bars, $500 \mathrm{~nm}$. Values are mean \pm standard deviation $(n=3) . * p<0.05$ versus all groups.

Abbreviations: EDC, I-ethyl-3-(3-dimethylaminopropyl) carbodiimide hydrochloride; ET6, biological tissue materials modified with EDC for 6 hours; GTA, glutaraldehyde; GTI, biological tissue materials modified with GTA for I hour; GT6, biological tissue materials modified with GTA for 6 hours; GT24, biological tissue materials modified with GTA for 24 hours. structure of collagen molecules. Sung et al have reported that the fixation of porcine pericardium with genipin and/ or carbodiimide causes contraction of the tropocollagen molecules and collagen networks, thereby contributing to the tissue shrinkage. ${ }^{29}$ In addition to genipin, GTA is another non-zero-length cross-linker that may produce a similar effect. The results of this study showed that during the treatment period from 1 to 24 hours, noticeably distinct collagen fibrillar structures of GTA cross-linked biological tissues are attributed to different extents of molecular aggregation. For the AM modified with longer time, the formation of large amount of interhelical cross-links may draw the collagen molecules closer together. Figure 2B further shows the results of quantitative analysis of nanofiber diameter. According to the earlier observations, the non-cross-linked AM is composed of collagen fibrils with diameter of around $50 \mathrm{~nm} .{ }^{9}$ For the samples from the ET6 groups, an average diameter of collagen fibers increased to $561 \pm 74 \mathrm{~nm}$. The nanofiber diameter in the GT1, GT6, and GT24 groups was $113 \pm 35,406 \pm 48$, and $920 \pm 89 \mathrm{~nm}$, respectively. There were significant differences among these groups $(P<0.05)$. Our findings suggest that the cross-linking treatment time may affect the nanoscale structure of AM matrices modified with GTA. Because the cells can sense nanometer dimensions, the chemical cross-linking-mediated alteration in fibrillar structure of collagenous tissue scaffolds is critical to determine their cell culture performances.

\section{In vitro degradability}

In this study, we considered the chemical cross-linking of AM matrices using GTA because of its high efficiency of collagenous biomaterials stabilization..$^{14}$ Because collagen nanofibers constitute the architectural framework of AM, the degradation test of this biological tissue material was performed under the action of collagenase. After 4 weeks of incubation of $\mathrm{AM}$ in the degradation medium at $37^{\circ} \mathrm{C}$, the residual mass percentage of various samples is shown in Figure 3. The non-cross-linked biological tissues were found to be completely degraded within 4 weeks. However, in the ET6 groups, approximately $20 \%$ of weight loss was noted, indicating that the carbodiimide cross-linking of AM is beneficial to resist collagenase degradation. The remaining weight in the GT6 groups was $63.7 \% \pm 2.5 \%$, which was significantly higher than those of the GT1 $(16.3 \% \pm 2.2 \%)$ groups $(P<0.05)$, but significantly lower than those of the GT24 $(95.9 \% \pm 3.2 \%)$ groups $(P<0.05)$. The variation of residual mass percentage follows the trend of the change in the crosslinking index during the course of GTA treatment (Figure 1). 


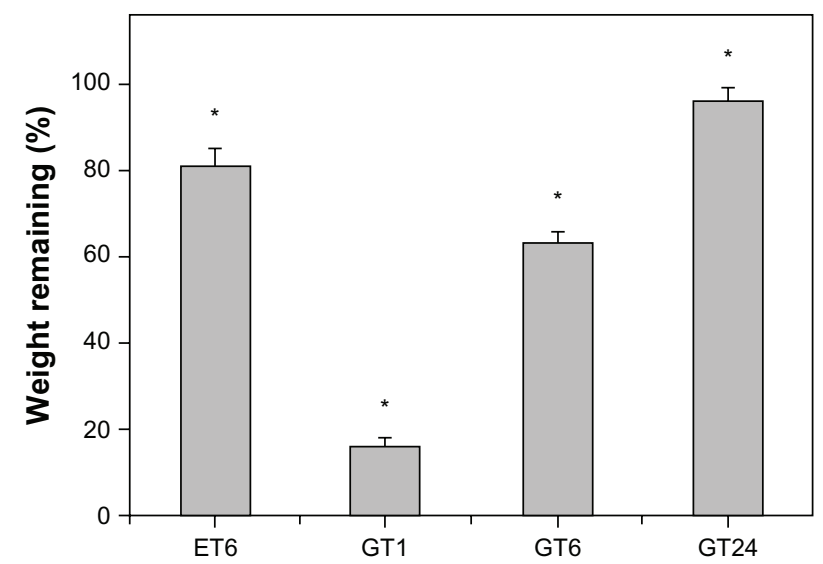

Figure 3 Weight remaining of various amniotic membrane samples after incubation at $37^{\circ} \mathrm{C}$ for 4 weeks in balanced salt solution containing collagenase.

Notes: Values are mean \pm standard deviation $(n=4)$. $* P<0.05$ versus all groups. Abbreviations: EDC, I-ethyl-3-(3-dimethylaminopropyl) carbodiimide hydrochloride; ET6, biological tissue materials modified with EDC for 6 hours; GTA, glutaraldehyde; GTI, biological tissue materials modified with GTA for I hour; GT6, biological tissue materials modified with GTA for 6 hours; GT24, biological tissue materials modified with GTA for 24 hours.

Given that the degradation of collagenous biomaterials is largely controlled by the collagenolytic cleavage of peptide bonds within the triple-helical structure, investigators have prepared GTA-fixed AM and introduced cross-links into these proteinaceous matrices to enhance the resistance against protease digestion. ${ }^{18,19}$ One possible explanation is that the high cross-linking can protect collagenase from approaching AM collagen molecules. Here, we further indicate that the newly formed linkages between the polypeptide chains may be responsible for the masking of specific enzymatic recognition sites by altering the matrix nanostructure of AM materials. The in vitro degradability of GTA cross-linked AM is possibly governed by collagen molecular aggregation driven by the cross-link content (Figure 2). Our data demonstrate that although the AM treated with carbodiimide for 24 hours has partial weight loss (about 17\%) after a 4-week degradation, the biological tissue counterparts from GT24 groups bridged by GTA remained intact without significant degradation.

\section{In vitro biocompatibility}

Biocompatibility is one of the major prerequisites for the development of functional biomaterials for potential ophthalmic applications. ${ }^{30}$ Because chemically modified AM are intended for use in ocular surface reconstruction, the interactions of biological tissue materials with corneal epithelial cells are studied in this paper. Figure 4A shows representative images of cells labeled with the Live/Dead stain. In the control groups, a bright green fluorescence was observed in HCE- 2 cell line cultures. In addition, only a few red-stained
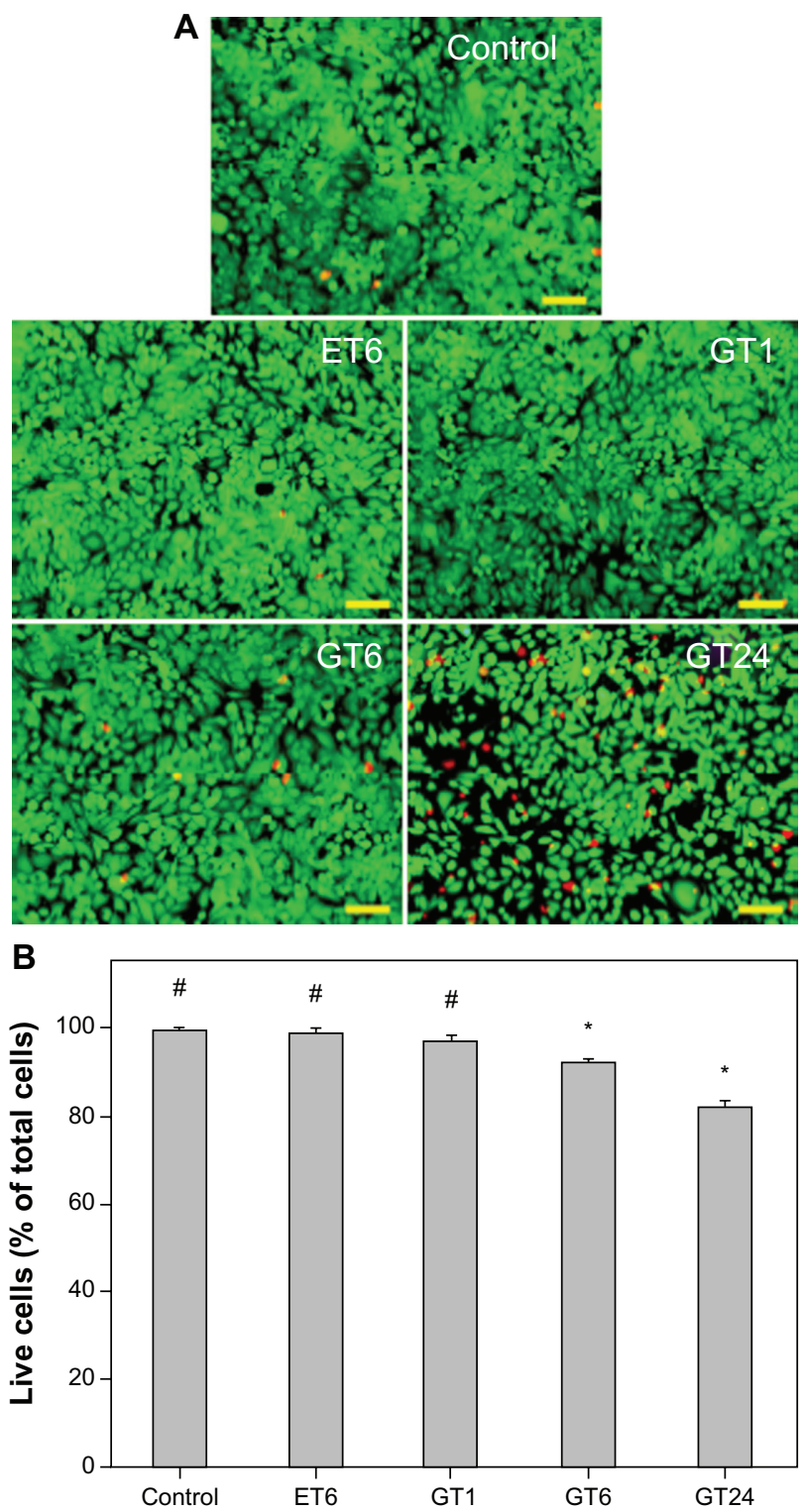

Figure 4 (A) Cell viability of human corneal epithelial cultures was determined by staining with Live/Dead Viability/Cytotoxicity Kit in which live cells fluoresce green and dead cells fluoresce red. Fluorescence images of cells after incubation for 3 days at $37^{\circ} \mathrm{C}$ with extract medium conditioned with various amniotic membrane samples. Control is without materials. (B) Mean percentage of live cells in the human corneal epithelial cultures as measured by the Live/Dead assay after a 3-day incubation with extract medium that had been treated with various amniotic membrane samples. Notes: Scale bars, $50 \mu \mathrm{m}$. Values are mean \pm standard deviation $(n=9)$. $* p<0.05$ versus all groups; ${ }^{\# P}<0.05$ versus GT6 and GT24 groups.

Abbreviations: EDC, I-ethyl-3-(3-dimethylaminopropyl) carbodiimide hydrochloride; ET6, biological tissue materials modified with EDC for 6 hours; GTA, glutaraldehyde; GTI, biological tissue materials modified with GTA for I hour; GT6, biological tissue materials modified with GTA for 6 hours; GT24, biological tissue materials modified with GTA for 24 hours.

nuclei were noted. These results indicate that the cells are mostly viable in the absence of test materials. In the ET6 and GT1 groups, the vast majority of HCE cell cultures were live. However, in the GT6 and GT24 groups, an increased number of red dots were seen. In particular, after 3 days of 
incubation with extract media that had been conditioned with sample GT24, the cultures contained a large amount of dead cells. Quantitative analysis of cell viability was performed after the Live/Dead assay, and the results are shown in Figure 4B. The mean percentage of live cells of control groups was $99.3 \% \pm 1.0 \%$. It was not significantly different from those of the ET6 $(98.9 \% \pm 1.2 \%)$ and GT1 $(97.6 \% \pm 0.9 \%)$ groups $(P>0.05)$, suggesting a large percentage of surviving HCE cells. The mean percentage of live cells of the GT6 and GT24 groups was significantly reduced by about 7\% and $17.5 \%$, respectively, compared with the control groups $(P<0.05)$. Our study showed HCE-2 cell damage caused by the exposure to these test articles.

The metabolic activity of the grown cells was determined by using the MTS assay. ${ }^{31}$ Figure 5 shows the results of HCE cell proliferation after 3 days of incubation with extract media that had been conditioned with various AM samples. Similar levels of mitochondrial dehydrogenase activity (MTS activity) were noted in the control and ET6 groups and were not statistically different $(P>0.05)$. Our data demonstrate that the EDC/NHS treated AM materials do not affect the proliferative capacity of the HCE- 2 cell line cultures. In contrast, the MTS activity in the GT1, GT6, and GT24 groups was 98.0\% $0.9 \%$, $93.6 \% \pm 1.3 \%$, and $80.4 \% \pm 2.2 \%$, respectively. There were significant differences among these groups $(P<0.05)$, suggesting that the biological tissues cross-linked with GTA for varied time durations may present different cell growth

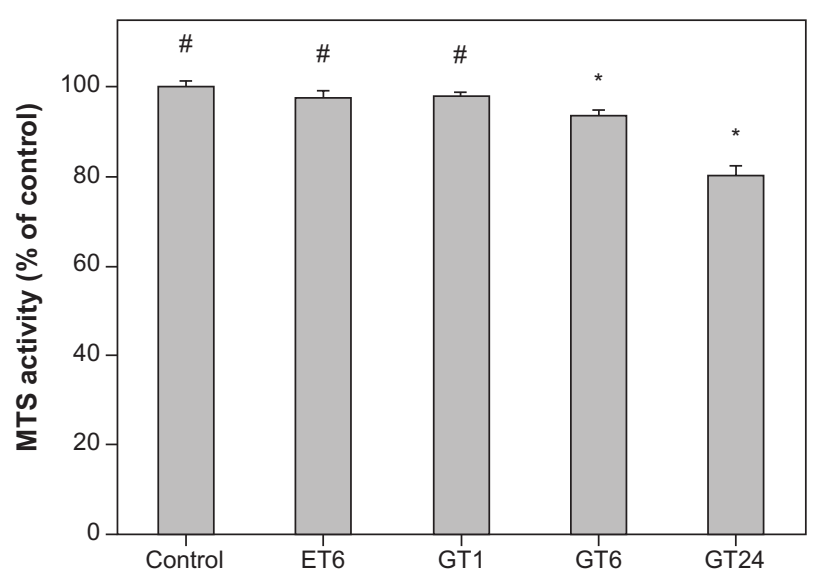

Figure 5 Cell proliferation assay of human corneal epithelial cultures incubated with extract medium conditioned with various amniotic membrane samples for 3 days. Notes: Results are expressed as percentage of controls (MTS activity of cells cultured in the absence of materials). Values are mean \pm standard deviation $(n=4)$. $* P<0.05$ versus all groups; ${ }^{*} P<0.05$ versus $\mathrm{GT} 6$ and $\mathrm{GT} 24$ groups.

Abbreviations: EDC, I-ethyl-3-(3-dimethylaminopropyl) carbodiimide hydrochloride; ET6, biological tissue materials modified with EDC for 6 hours; GTA, glutaraldehyde; GTI, biological tissue materials modified with GTA for I hour; GT6, biological tissue materials modified with GTA for 6 hours; GT24, biological tissue materials modified with GTA for 24 hours; MTS, 3-(4,5-dimethylthiazol-2-yl)-5-(3-carboxymethoxyphenyl)-2-(4-sulfophenyl)-2H-tetrazolium, inner salt. patterns. The inhibition of HCE cell proliferation is evident after 3 days of incubation with extract media that had been conditioned with sample GT24.

$I L-6$ has been considered to be involved in corneal grafting. ${ }^{32}$ Therefore, the $I L-6$ mRNA levels were quantified here by real-time RT-PCR. Figure 6 shows the proinflammatory gene expression of HCE-2 cell line cultures after 3 days of incubation with extract media that had been conditioned with various AM samples. No significant difference was observed in the $I L-6$ gene expression between the control and ET6 groups $(P>0.05)$, indicating that the carbodiimide crosslinked AM materials do not induce the pro-inflammatory response in HCE cells. The expression level was significantly higher in the GT24 (390.3\% $36.0 \%)$ groups compared with those of the GT1 (126.3\% $\pm 18.1 \%)$ and GT6 (224.0\% $\pm 29.2 \%)$ groups $(P<0.05)$. The finding suggests that the exposure of corneal epithelial cells to extract medium conditioned with sample GT24 may enhance the mRNA expression of $I L-6$.

Despite its ability to improve material stability, the chemical treatment with cross-linker probably causes toxic effects. Hence, we examine the biocompatibility of chemically modified AM materials by cell viability and proliferation assays and proinflammatory gene expression analyses. Results of this study show that the EDC/NHS cross-linked AM has good compatibility toward HCE cells. Given

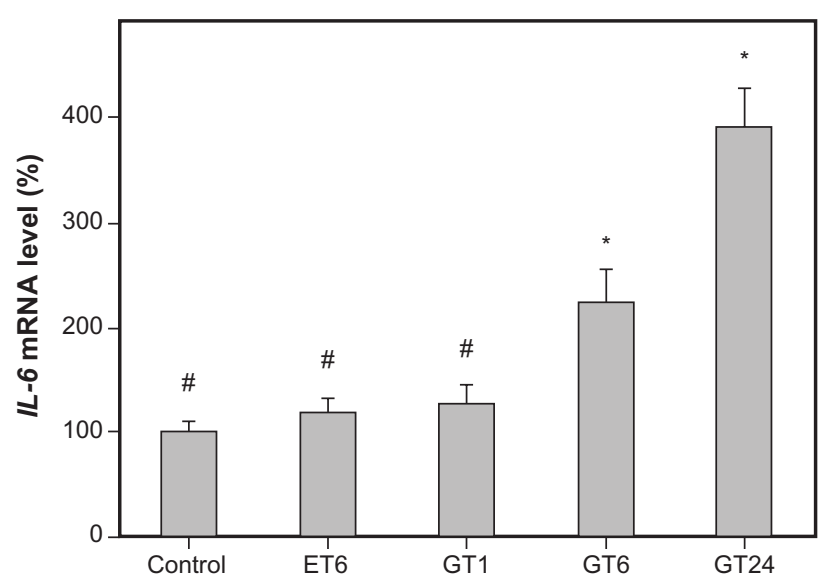

Figure 6 Gene expression of IL-6 in human corneal epithelial cells incubated with extract medium conditioned with various amniotic membrane samples for 3 days, measured by real-time RT-PCR.

Notes: Normalization was done by using GAPDH. Data in the experimental groups are percentages relative to those of control groups (without materials). Values are mean \pm standard deviation $(n=3)$. ${ }^{* P}<0.05$ versus all groups; ${ }^{\#}<0.05$ versus GT6 and GT24 groups.

Abbreviations: EDC, I-ethyl-3-(3-dimethylaminopropyl) carbodiimide hydrochloride; ET6, biological tissue materials modified with EDC for 6 hours; GTA, glutaraldehyde; GTI, biological tissue materials modified with GTA for I hour; GT6, biological tissue materials modified with GTA for 6 hours; GT24, biological tissue materials modified with GTA for 24 hours; IL-6, interleukin-6; RT-PCR, reverse transcription-polymerase chain reaction; GAPDH, glyceraldehyde-3phosphate dehydrogenase; mRNA, messenger RNA. 
the safety of carbodiimide chemistry, this modification technique has been successfully applied in the synthesis of aminated gelatin grafted with carboxylic end-capped $\operatorname{poly}(N$ isopropylacrylamide) to fabricate biodegradable in situ gelling drug delivery system. ${ }^{23}$ In contrast, the samples cross-linked with GTA for 1 hour are not detrimental to the HCE- 2 cell cultures. With increasing GTA treatment time to 6 hours, the cross-linked AM causes mild cytotoxicity. Nevertheless, the viability of corneal epithelial cells exposed to biological tissues of GT6 groups is found to be more than $90 \%$, indicating acceptable biocompatibility. We have previously shown that because of the presence of Michael-type adducts with terminal aldehydes, the GTA cross-linked gelatin ${ }^{16}$ and chitosan $^{33}$ materials are not well-tolerated by the ocular cell cultures. Although the aldehyde groups introduced in the AM matrices are quenched with glycine here, the samples treated with GTA for a longer duration (ie, 24 hours) may result in more intense toxicity and inflammation. This is probably a result of the formation of abundant Michael-type adducts with terminal aldehydes in the cross-linking structure of AM materials having a greater extent of cross-linking. The decrease in biocompatibility is attributable to the interaction between the exposed aldehyde groups with the surface of cultured cells. ${ }^{13}$ The information from GTA treated AM-HCE cell interactions that are relevant to in vitro biocompatibility is rarely found and will be valuable for biomedical research.

\section{Anti-inflammatory activity}

TNF- $\alpha$ is one of the major cytokine-triggering mediators of inflammatory reactions. It has been reported that the activity of proinflammatory cytokine such as TNF- $\alpha$ should be regulated to prevent uncontrolled inflammation. ${ }^{34}$ Human AM tissues have anti-inflammatory properties and are effective in reducing inflammation after transplantation. ${ }^{20}$ In this work, the anti-inflammatory actions of chemically modified AM materials were studied. Figure 7 shows the expression levels of secreted TNF- $\alpha$ after incubation of HCE cells with various AM samples for 3 days. The measured concentration of TNF- $\alpha$ in the ET6 groups was $608.1 \pm 30.7 \mathrm{pg} / \mathrm{mL}$. It was significantly higher than those of the $\mathrm{NC}(79.6 \pm 25.2 \mathrm{pg} / \mathrm{mL})$ groups but significantly lower than those of the PC $(1804.7 \pm 51.1 \mathrm{pg} / \mathrm{mL})$ groups $(P<0.05)$. These observations indicate that LPS can upregulate the production of TNF- $\alpha$ in HCE-2 cell cultures. In addition, the carbodiimide cross-linked AM materials still have anti-inflammatory effects. In contrast, the measured concentration of TNF- $\alpha$ in the GT1, GT6, and GT24 groups was $654.3 \pm 28.4,747.0 \pm 37.1$, and $865.7 \pm 40.4 \mathrm{pg} / \mathrm{mL}$, respectively. There were significant dif-

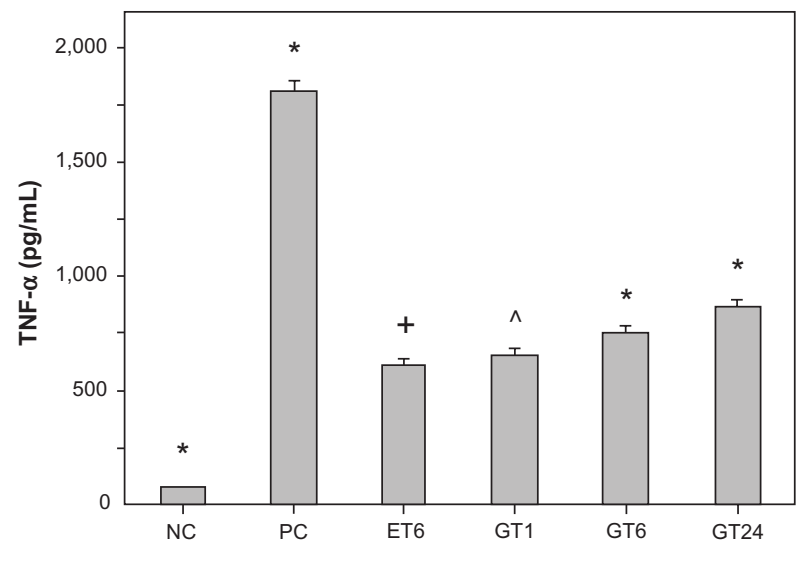

Figure 7 Level of TNF- $\alpha$ released from human corneal epithelial cell line cultures after incubation with various amniotic membrane samples for 3 days.

Notes: Unstimulated and LPS-stimulated cells without contacting the test materials were the NC and PC groups, respectively. Values are mean \pm standard deviation $(n=4) . * P<0.05$ versus all groups; ${ }^{+} P<0.05$ versus all groups, except $G T I ;{ }^{\wedge} P<0.05$ versus all groups, except ET6.

Abbreviations: EDC, I-ethyl-3-(3-dimethylaminopropyl) carbodiimide hydrochloride; ET6, biological tissue materials modified with EDC for 6 hours; GTA, glutaraldehyde; GTI, biological tissue materials modified with GTA for I hour; GT6, biological tissue materials modified with GTA for 6 hours; GT24, biological tissue materials modified with GTA for 24 hours; TNF- $\alpha$, tumor necrosis factor- $\alpha$; LPS, lipopolysaccharide; $\mathrm{NC}$, negative control; PC, positive control.

ferences among these groups $(P<0.05)$, suggesting that the anti-inflammatory activity of AM is highly correlated with the extent of cross-linking of GTA treated biological tissue. As reported in the literature, the anti-inflammatory properties of AM materials strongly depend on the proteins expressed in mesenchymal cells. ${ }^{35}$ One possible explanation for our observations is that the amount of new cross-links formed between the collagen nanofibers plays an important role in the modulation of protein structure, thereby affecting the expression of TNF- $\alpha$ and anti-inflammatory action of AM. To the best of our knowledge, the relationship between the cross-linking chemistry-mediated nanoscale structure and anti-inflammatory activity of GTA-treated AM tissue has not been documented elsewhere.

\section{Stemness gene expression}

It is generally believed that the development of a culture platform for supporting the survival of epithelial progenitor cells will assist scientists in applying this tissue engineering technique for ocular surface reconstruction. ${ }^{36}$ Biomaterials have been used to tailor the biophysical and biochemical properties of the culture microenvironment that allows stem cell self-renewal. ${ }^{37}$ Therefore, the effect of nanofibrous structure of chemically modified proteinaceous matrices on the maintenance of cell stemness (ie, undifferentiated state) was investigated to give insight into the limbal stem cell niche. Figure 8 shows the $A B C G 2$ gene expression of 
rabbit LECs after growth on various AM samples for 5 days. By using the techniques of quantitative real-time RT-PCR, the mRNA level in the control groups (ie, absence of AM materials) was defined as $100 \%$. It was significantly lower than those of the non-cross-linked AM $(181.6 \% \pm 28.4 \%)$ and all the cross-linked AM groups $(P<0.05)$, indicating that these biological tissues are effective to preserve the progenitor cells. In corneal tissue engineering, the AM has been used as a niche for ex vivo expansion of limbal stem cells because this collagenous tissue material helps to retain stemness in the cells. ${ }^{6}$ Our findings are compatible with this evidence and suggest that the chemical cross-linking treatment does not hinder the ability of AM to preserve the progenitors of LECs in vitro. Interestingly, the $A B C G 2$ expression in the ET6, GT1, GT6, and GT24 groups was $896.9 \% \pm 47.0 \%$, $254.4 \% \pm 35.8 \%, 683.0 \% \pm 32.1 \%$, and $1,397.8 \% \pm 59.2 \%$, respectively. These values showed statistically significant differences $(P<0.05)$, demonstrating that the LEC cultures on the chemically modified AM samples have varying levels of enhanced stemness.

More recently, Sekar et al have evaluated the efficacy of $\mathrm{Al}_{2}\left(\mathrm{SO}_{4}\right)_{3}$ cross-linked $\mathrm{AM}$ for ocular surface reconstruction. ${ }^{38}$ The cross-linked biological tissue exhibits improved mechanical properties and shows its feasibility to be used for cultivation of limbal epithelial sheets. GTA is one of the most commonly reported chemical cross-linkers

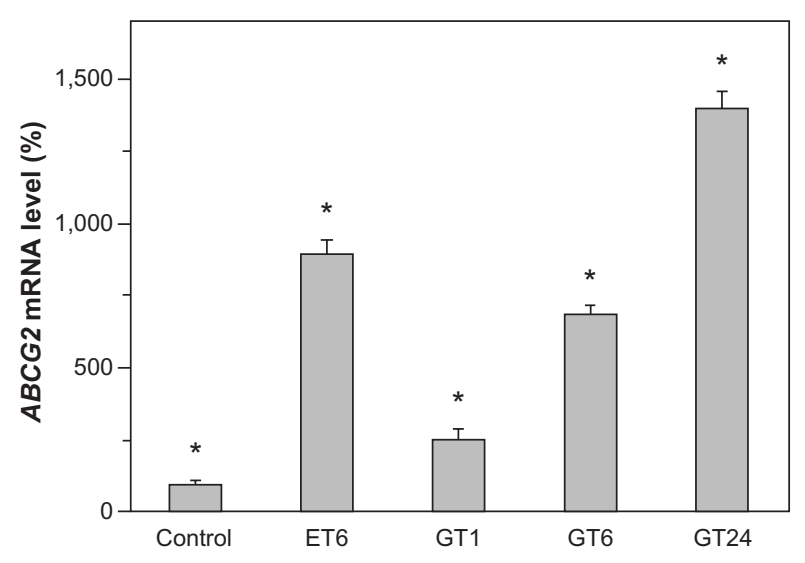

Figure 8 Gene expression of $A B C G 2$ in rabbit LECs grown on various AM samples for 5 days, measured by real-time RT-PCR. Normalization was done by using GAPDH. Data in the experimental groups are percentages relative to those of control groups (cells cultured on TCPS in the absence of AM materials). Values are mean \pm standard deviation $(n=3)$. $* P<0.05$ versus all groups.

Abbreviations: ABCG2, ATP binding cassette, subfamily G, member 2; ET6, biological tissue materials modified with EDC for 6 hours; GAPDH, glyceraldehyde3-phosphate dehydrogenase; GTA, glutaraldehyde; GTI, biological tissue materials modified with GTA for I hour; GT6, biological tissue materials modified with GTA for 6 hours; GT24, biological tissue materials modified with GTA for 24 hours; mRNA, messenger RNA; LEC, limbal epithelial cell; AM, amniotic membrane; RT-PCR, reverse transcription polymerase chain reaction; TCPS, tissue culture polystyrene. featured with high efficiency for collagenous biomaterials stabilization, making it attractive for application in the modification of AM matrices. Given that the cells can sense nanometer dimensions, the observed differences in stemness gene expression probably reflect the critical role of nanostructure of chemically cross-linked AM collagen molecules. Recently, poly( $\varepsilon$-caprolactone $)^{39}$ or poly(D, L-lactide-co-glycolide $)^{40}$ nanofibrous scaffolds have been developed that provide a platform for cell differentiation. Yang et al have further examined the efficacy of poly(L-lactic acid) nano/micro fibrous scaffolds for neural tissue engineering and found that the neural stem cell differentiation rate was higher for nanofibers than that of microfibers. ${ }^{41}$ To investigate the effect of fiber size on osteoblastic MG63 cellmaterial interactions, Sisson et al have fabricated electrospun scaffolds consisting of gelatin nanofibers with diameters ranging from 110 to $600 \mathrm{~nm} .{ }^{42}$ Their study suggests that the small-fiber-diameter scaffold is better for cell differentiation than its large-fiber-diameter counterparts. In light of these facts, increasing nanofiber diameter may facilitate the maintenance of the undifferentiated precursor cell phenotype. It possibly explains the highest expression of stem cell marker $A B C G 2$ for the LECs on the sample GT24. However, the exact mechanism remains unclear, and further research is required to define the precise role of collagen molecular aggregation in the determination of cell fate in response to the chemically modified AM materials. In contrast, to confirm the maintenance of undifferentiated progenitor cell population on intact and denuded AM matrices, Sudha et al have reported that at least two markers of progenitor cells must be used in addition to two markers of differentiation. ${ }^{43}$ Here, we simply quantify one gene product to evaluate the stemness expression levels. Another limitation of the current study is that we do not check the expressions of corneal differentiation markers.

\section{Conclusion}

In this work, we have evaluated the use of GTA for the chemical modification of AM materials. At the reaction time of 6 hours, the cross-linking degree and in vitro degradability of GTA-treated samples are much lower than those of the carbodiimide cross-linked counterparts. In addition, the lower extent of aggregation of collagen nanofibers in the GTA cross-linked biological tissues is probably a result of the less-efficient penetration of cross-linkers into the proteinaceous matrices. However, the inverse results are obtained at the treatment for 24 hours, indicating that the GTA is a powerful cross-linking agent to improve the 
stability of AM. As demonstrated by the in vitro biocompatibility studies, the samples cross-linked with GTA for 24 hours are not well-tolerated by the HCE cell cultures. For the GTA-treated AM groups, the cross-linking efficiency is increased with increasing reaction time. In particular, when the treatment duration is less than 6 hours, the biological tissues cross-linked with GTA for longer time may cause slight reductions in MTS and anti-inflammatory activities. Nevertheless, significant collagen molecular aggregation also enhances the ability of these AM matrices to preserve the progenitors of LECs in vitro. For the first time, here we demonstrate that the GTA cross-linking of AM materials affects their nanofibrous structures and cell culture characteristics. Our future interest will be toward more fully exploring the potential of AM cross-linked with GTA for 6 hours as a carrier for delivery of cultured LECs to the ocular surface.

\section{Acknowledgment}

The authors are grateful to Ya-Ting Li (Institute of Biochemical and Biomedical Engineering, Chang Gung University) for technical assistance. This work was supported by grant NSC102-2628-B-182-017-MY3 from the National Science Council of Republic of China and grant CMRPD2C0071 from Chang Gung Memorial Hospital.

\section{Disclosure}

The authors report no conflicts of interest in this work.

\section{References}

1. Langer R, Vacanti JP. Tissue engineering. Science. 1993;260(5110): 920-926.

2. Gris O, López-Navidad A, Caballero F, del Campo Z, Adán A. Amniotic membrane transplantation for ocular surface pathology: long-term results. Transplant Proc. 2003;35(5):2031-2035.

3. Riau AK, Beuerman RW, Lim LS, Mehta JS. Preservation, sterilization and de-epithelialization of human amniotic membrane for use in ocular surface reconstruction. Biomaterials. 2010;31(2):216-225.

4. Iijima K, Igawa Y, Imamura T, et al. Transplantation of preserved human amniotic membrane for bladder augmentation in rats. Tissue Eng. 2007;13(3):513-524.

5. Yang L, Shirakata Y, Tokumaru S, et al. Living skin equivalents constructed using human amnions as a matrix. J Dermatol Sci. 2009;56(3): 188-195.

6. Grueterich M, Espana EM, Tseng SC. Ex vivo expansion of limbal epithelial stem cells: amniotic membrane serving as a stem cell niche. Surv Ophthalmol. 2003;48(6):631-646.

7. Tsai RJ, Li LM, Chen JK. Reconstruction of damaged corneas by transplantation of autologous limbal epithelial cells. $N$ Engl J Med. 2000;343(2):86-93.

8. Slansky HH, Dohlman CH. Collagenase and the cornea. Surv Ophthalmol. 1970;14(5):402-415.

9. Ma DH, Lai JY, Cheng HY, Tsai CC, Yeh LK. Carbodiimide crosslinked amniotic membranes for cultivation of limbal epithelial cells. Biomaterials. 2010;31(25):6647-6658.
10. Lai JY, LiYT. Influence of cross-linker concentration on the functionality of carbodiimide cross-linked gelatin membranes for retinal sheet carriers. J Biomater Sci Polym Ed. 2011;22(1-3):277-295.

11. Jayakrishnan A, Jameela SR. Glutaraldehyde as a fixative in bioprostheses and drug delivery matrices. Biomaterials. 1996;17(5):471-484.

12. Lai JY, Ma DH, Cheng HY, et al. Ocular biocompatibility of carbodiimide cross-linked hyaluronic acid hydrogels for cell sheet delivery carriers. J Biomater Sci Polym Ed. 2010;21(3):359-376.

13. Lai JY. Biocompatibility of chemically cross-linked gelatin hydrogels for ophthalmic use. J Mater Sci Mater Med. 2010;21(6):1899-1911.

14. Bigi A, Cojazzi G, Panzavolta S, Rubini K, Roveri N. Mechanical and thermal properties of gelatin films at different degrees of glutaraldehyde crosslinking. Biomaterials. 2001;22(8):763-768.

15. Matsuda S, Iwata H, Se N, Ikada Y. Bioadhesion of gelatin films crosslinked with glutaraldehyde. J Biomed Mater Res. 1999;45(1):20-27.

16. Lai JY, Li YT. Evaluation of cross-linked gelatin membranes as delivery carriers for retinal sheets. Mater Sci Eng C, Biomim Supramol Syst. 2010;30(5):677-685.

17. Olde Damink LH, Dijkstra PJ, van Luyn MJ, van Wachem PB, Nieuwenhuis P, Feijen J. Cross-linking of dermal sheep collagen using a water-soluble carbodiimide. Biomaterials. 1996;17(8):765-773.

18. Fujisato T, Tomihata K, Tabata Y, Iwamoto Y, Burczak K, Ikada Y. Cross-linking of amniotic membranes. J Biomater Sci Polym Ed. 1999;10(11):1171-1181.

19. Spoerl E, Wollensak G, Reber F, Pillunat L. Cross-linking of human amniotic membrane by glutaraldehyde. Ophthalmic Res. 2004;36(2): 71-77.

20. Lu PL, Lai JY, Tabata Y, Hsiue GH. A methodology based on the "anterior chamber of rabbit eyes" model for noninvasively determining the biocompatibility of biomaterials in an immune privileged site. J Biomed Mater Res A. 2008;86(1):108-116.

21. Lai JY, LiYT, Cho CH, Yu TC. Nanoscale modification of porous gelatin scaffolds with chondroitin sulfate for corneal stromal tissue engineering. Int J Nanomedicine. 2012;7:1101-1114.

22. Lai JY, Chen KH, Hsiue GH. Tissue-engineered human corneal endothelial cell sheet transplantation in a rabbit model using functional biomaterials. Transplantation. 2007;84(10):1222-1232.

23. Lai JY, Hsieh AC. A gelatin-g-poly(N-isopropylacrylamide) biodegradable in situ gelling delivery system for the intracameral administration of pilocarpine. Biomaterials. 2012;33(7):2372-2387.

24. Lai JY, Wang TP, Li YT, Tu IH. Synthesis, characterization and ocular biocompatibility of potential keratoprosthetic hydrogels based on photopolymerized poly(2-hydroxyethyl methacrylate)-co-poly(acrylic acid). J Mater Chem. 2012;22(5):1812-1823.

25. Kim DH, Novak MT, Wilkins J, Kim M, Sawyer A, Reichert WM. Response of monocytes exposed to phagocytosable particles and discs of comparable surface roughness. Biomaterials. 2007;28(29): 4231-4239.

26. Lai JY. Evaluation of cross-linking time for porous gelatin hydrogels on cell sheet delivery performance. J Mech Med Biol. 2011;11(5):967-981.

27. Gough JE, Scotchford CA, Downes S. Cytotoxicity of glutaraldehyde crosslinked collagen/poly(vinyl alcohol) films is by the mechanism of apoptosis. J Biomed Mater Res. 2002;61(1):121-130.

28. Franchi M, Trirè A, Quaranta M, Orsini E, Ottani V. Collagen structure of tendon relates to function. Scientific World Journal. 2007;7: 404-420.

29. Sung HW, Chang WH, Ma CY, Lee MH. Crosslinking of biological tissues using genipin and/or carbodiimide. J Biomed Mater Res A. 2003;64(3):427-438.

30. Lai JY. Biocompatibility of genipin and glutaraldehyde cross-linked chitosan materials in the anterior chamber of the eye. Int J Mol Sci. 2012;13(9):10970-10985.

31. Lu PL, Lai JY, Ma DH, Hsiue GH. Carbodiimide cross-linked hyaluronic acid hydrogels as cell sheet delivery vehicles: characterization and interaction with corneal endothelial cells. J Biomater Sci Polym Ed. 2008;19(1):1-18. 
32. Ventura AC, Böhnke M. Pentoxifylline influences the autocrine function of organ cultured donor corneas and enhances endothelial cell survival. Br J Ophthalmol. 2001;85(9):1110-1114.

33. Lai JY, Li YT, Wang TP. In vitro response of retinal pigment epithelial cells exposed to chitosan materials prepared with different cross-linkers. Int J Mol Sci. 2010;11(12):5256-5272.

34. He H, Li W, Chen SY, et al. Suppression of activation and induction of apoptosis in RAW264.7 cells by amniotic membrane extract. Invest Ophthalmol Vis Sci. 2008;49(10):4468-4475.

35. Hao Y, Ma DH, Hwang DG, Kim WS, Zhang F. Identification of antiangiogenic and anti-inflammatory proteins in human amniotic membrane. Cornea. 2000;19(3):348-352.

36. Ma DH, Chen HC, Lai JY, et al. Matrix revolution: molecular mechanism for inflammatory corneal neovascularization and restoration of corneal avascularity by epithelial stem cell transplantation. Ocul Surf. 2009;7(3):128-144.

37. Bratt-Leal AM, Carpenedo RL, Ungrin MD, Zandstra PW, McDevitt TC. Incorporation of biomaterials in multicellular aggregates modulates pluripotent stem cell differentiation. Biomaterials. 2011;32(1): $48-56$.
38. Sekar S, Sasirekha K, Krishnakumar S, Sastry TP. A novel cross-linked human amniotic membrane for corneal implantations. Proc Inst Mech Eng H. 2013;227(3):221-228.

39. Nirmala R, Kang HS, Park HM, Navamathavan R, Jeong IS, Kim HY. Silver-loaded biomimetic hydroxyapatite grafted poly(epsiloncaprolactone) composite nanofibers: a cytotoxicity study. $J$ Biomed Nanotechnol. 2012;8(1):125-132.

40. Zanatta G, Rudisile M, Camassola M, et al. Mesenchymal stem cell adherence on poly(D, L-lactide-co-glycolide) nanofibers scaffold is integrin-beta 1 receptor dependent. J Biomed Nanotechnol. 2012;8(2): 211-218.

41. Yang F, Murugan R, Wang S, Ramakrishna S. Electrospinning of nano/ micro scale poly(L-lactic acid) aligned fibers and their potential in neural tissue engineering. Biomaterials. 2005;26(15):2603-2610.

42. Sisson K, Zhang C, Farach-Carson MC, Chase DB, Rabolt JF. Fiber diameters control osteoblastic cell migration and differentiation in electrospun gelatin. J Biomed Mater Res A. 2010;94(4):1312-1320.

43. Sudha B, Sitalakshmi G, Iyer GK, Krishnakumar S. Putative stem cell markers in limbal epithelial cells cultured on intact and denuded human amniotic membrane. Indian J Med Res. 2008;128(2):149-156.
International Journal of Nanomedicine

\section{Publish your work in this journal}

The International Journal of Nanomedicine is an international, peerreviewed journal focusing on the application of nanotechnology in diagnostics, therapeutics, and drug delivery systems throughout the biomedical field. This journal is indexed on PubMed Central, MedLine, CAS, SciSearch $\AA$, Current Contents ${ }^{\circledR} /$ Clinical Medicine,

\section{Dovepress}

Journal Citation Reports/Science Edition, EMBase, Scopus and the Elsevier Bibliographic databases. The manuscript management system is completely online and includes a very quick and fair peer-review system, which is all easy to use. Visit http://www.dovepress.com/ testimonials.php to read real quotes from published authors. 